\title{
Uso de vasoconstritores em pacientes portadores de Hipertireoidismo
}

\author{
Use of vasoconstrictors in patients with Hyperthyroidism \\ Uso de vasoconstrictores em pacientes portadores de Hipertiroidismo
}

\section{Emanuele Castro de Araujo ${ }^{1 *}$, Thaís Evellyn da Silva ${ }^{2}$, Marcela Paschoal Rocha ${ }^{3}$, Leandro Moraes Coelho de Oliveira ${ }^{4}$}

Como citar esse artigo. de Araujo, EC; da Silva, TE; Rocha, MP; de Oliveira, LMC. Uso de vasoconstritores em pacientesportadoresdeHipertireoidismo. Revista Pró-UniverSUS. 2019 Jan./Jun.; 10 (1): $133-136$.

\begin{abstract}
Resumo
Através da anestésica local é possível a realização de grande parte dos procedimentos odontológicos. Os anestésicos locais são substâncias que bloqueiam a condução nervosa, causando a perda temporária da sensibilidade de regiões do corpo. O uso do vasoconstritor traz benefícios para os anestésicos, como a absorção lenta do sal anestésico que resulta em um efeito mais prolongado da anestesia local. Entretanto, em pacientes com determinadas condições sistêmicas, precisam ser adequadamente indicados. É o caso de pacientes portadores de hipertireoidismo, que são mais sensíveis aos vasoconstritores. Foram realizadas buscas em bases de dados Scientific Eletronic Library Online (SciELO), Google Acadêmico e PubMed com os descritores: "Hipertireoidismo", "vasoconstritor", "anestésicos locais" e "alterações sistêmicas" além da utilização dos livros de terapêutica medicamentosa e urgências médicas em odontologia, no período de novembro a dezembro de 2018. Uma seleção de 14 documentos serviu de referência bibliográfica para a construção desta revisão de literatura. O objetivo dessa revisão de literatura é abordar o uso de vasoconstritores em pacientes com hipertireoidismo de modo que, o profissional possa intervir com segurança em procedimentos odontológicos que requeiram anestesia local. Foi concluído que, utilizar os vasoconstritores ou não nos procedimentos odontológicos em pacientes com hipertireoidismo, deve ser bem analisado levando em consideração a sua condição sistêmica prévia e atual.

Palavras-chave: Anestesia local; Vasoconstritores; Hipertireoidismo.
\end{abstract}

\begin{abstract}
Through local anesthetic it is possible to carry out most of the dental procedures. Local anesthetics are substances that block nerve conduction, causing temporary loss of sensitivity of regions of the body. The use of the vasoconstrictor provides benefits for anesthetics, such as slow absorption of anesthetic salt resulting in a prolonged effect of local anesthesia. However, in patients with certain systemic conditions, they must be adequately indicated. It isthe case of patients with hyperthyroidism, which are more sensitive to vasoconstrictors. Searches were carried out in data bases Scientific Eletronic Library Online (SciELO), Academic Google and PubMed with the descriptors: "Hyperthyroidism", "vasoconstrictor", "local anesthetics" and "systemic alterations" besides the use of medical therapy books and medical emergencies in dentistry, in the period from novemberto december 2018. A selection of 14 bibliographic reference documents for the construction of the review literature. The purpose of this literature review is to address the use of vasoconstrictors in patients with hyperthyroidism so that the professional can safely intervene in dental procedures that require local anestesia. It was concluded that using vasoconstrictors or not in dental procedures in patients with hyperthyroidism should be well analyzed taking into account their previous and current systemic condition.
\end{abstract}

Keywords: Local Anesthesia; Vasoconstrictors; Hyperthyroidism.

\section{Resumen}

Através de la anestésica local es posible la realización de gran parte de los procedimientos odontológicos. Los anestésicos locales son sustancias que bloquean la conducción nerviosa, causando la pérdida temporal de la sensibilidad de las regiones del cuerpo. El uso del vasoconstrictor trae beneficios para los anestésicos, como la absorción lenta de la sal anestésica que resulta en un efecto más prolongado de la anestesia local. Sin embargo, en pacientes con ciertas condiciones sistémicas, deben ser adecuadamente indicados. Es el caso de pacientes portadores de hipertiroidismo, que son más sensibles a los vasoconstrictores. Se realizaron búsquedas en bases de datos Scientific Eletronic Library Online (SciELO), Google Académico y PubMed con los descriptores: "Hipertiroidismo", "vasoconstrictor", "anestésicos locales" y "alteraciones sistémicas" además de la utilización de los libros de terapia medicamentosa y urgencias médicos en odontología, en el período de noviembre a diciembre de 2018. Una selección de 14 documentos sirvió de referencia bibliográfica para la construcción de esta revisión de literatura. El objetivo de esta revisión de literatura es abordar el uso de vasoconstrictores en pacientes con hipertiroidismo de modo que el profesional pueda intervenir con seguridad en procedimientos odontológicos que requieran anestesia local. Se concluyó que, utilizar los vasoconstrictores o no en los procedimientos odontológicos en pacientes con hipertiroidismo, debe ser bien analizado teniendo en cuenta su condición sistémica previa y actual.

Palabras clave: Anestesia Local; Vasoconstrictores; Hipertiroidismo.

Afiliação dos autores:

1Cirurgiã-Dentista graduada pela Universidade de Vassouras. RJ, Brasil. Email: emanuelecaraujo96@gmail.com ORCID: https://orcid.org/0000-0002-4524-1102

2 Cirurgiã-Dentista graduada pela Universidade de Vassouras. RJ, Brasil. Email: thais-evellyn@hotmail.com ORCID: https://orcid.org/0000-0002-0547-6222

3Cirurgiã-Dentista graduada pela Universidade de Vassouras. RJ, Brasil. Email: marcela.procha@hotmail.com ORCID: https://orcid.org/0000-0002-0519-7979

4Mestrando em Saúde Coletiva pela São Leopoldo Mandic, Campinas. SP Brasil. Email: leandromcoliveira@hotmail.com ORCID: https://orcid.org/0000-0003-0944-8445

* Email de correspondencia: emanuelecaraujo96@gmail.com 


\section{Introdução}

Os anestésicos locais são substâncias que em contato com as fibras nervosas resultam em um bloqueio reversível da condução nervosa, interrompendo todas as modalidades de influxo nervoso. Com isso, promovem

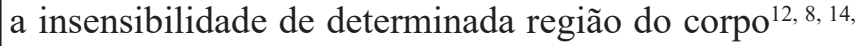
$3,1$.

Desde a antiguidade, vem se buscando substâncias que pudessem diminuir a dor dos pacientes. O ópio, suco da Papaversomniferum, de nome popular papoula, com suas propriedades hipnóticas e sedativas, era utilizado. Também eram utilizados asfixia temporária, que causava um desmaio momentâneo e se necessário, uma pancada na cabeça do paciente. E se ainda assim, não tivesse a resposta esperada, o paciente era imobilizado por quatro auxiliares e o cirurgião realizava o procedimento ${ }^{8,4}$.

A cocaína foi o primeiro anestésico local utilizado na Medicina e Odontologia ${ }^{8,12}$. Seus benefícios foram estudados, e posteriormente passou a ser administrada com eficácia em procedimentos odontológicos. ${ }^{8}$ Outros anestésicos, utilizados até hoje, surgiram a partir da procura de substitutos para a cocaína ${ }^{8,12}$.

Para obter uma anestesia local segura e eficaz, o cirurgião-dentista deve conhecer e selecionar corretamente o fármaco mais apropriado para cada paciente segundo sua condição sistêmica ${ }^{1,7}$.

Os anestésicos locais se dividem em éster e amida. Os mais utilizados na odontologia são a lidocaína e prilocaína do grupo amida, pois são uma alternativa menos tóxica e mais efetiva ${ }^{14}$.

Os primeiros anestésicos a serem utilizados foram do grupo éster. Porém, devido a sua alta toxicidade não são comercializados no Brasil. São eles a cocaína, procaína, cloroprocaína, tetracaína e benzocaína. A benzocaína é comumente utilizada como anestésico de superfície ${ }^{14,1}$. Já os anestésicos do grupo amida são mais estáveis, raramente ocorrem reações alérgicas e tem maior duração de ação. Como exemplo citamse a lidocaína, prilocaína, mepivacaína, bupivacaína, articaína, etidocaína e ropivacaína ${ }^{12,1}$. A ropivacaína e a etidocaína não estão disponíveis em tubetes para uso odontológico no Brasil ${ }^{1}$.

Um fármaco, para ser considerado um bom agente anestésico, deve ser não irritante, não causar lesões aos tecidos, ter baixa toxicidade sistêmica, tempo de início de ação rápida e duração suficiente para realizar o procedimento, propriedades de penetração suficiente para ser anestésico tópico, ser livre de reações alérgicas e estar estéril ou capaz de ser esterilizado sem deterioração ${ }^{8,11}$. Mas, nenhum anestésico alcança todas essas propriedades ${ }^{11}$.

Todos as soluções anestésicas possuem ações vasodilatadoras $^{9,8,1}$. Quando são injetadas próximos das fibras e troncos nervosos, a dilatação dos capilares sanguíneos proporciona sua rápida absorção, limitando o tempo de ação do anestésico ${ }^{1}$. O que explica a associação do vasoconstritor nas substâncias anestésicas. Essa interação farmacológica aumenta o tempo de ação do sal anestésico em contato com as fibras. Isso proporciona um tempo de ação maior e efeito mais eficaz, reduzindo o grau de toxicidade devido a uma menor quantidade de anestésico a ser utilizada ${ }^{9,12,11,2,1}$.

Injetados, os anestésicos desenvolvem suas ações clínicas. Após isso, são reabsorvidos, e na corrente sanguínea se distribuem por todos os compartilhamentos. Diante disso, o sistema nervoso central (SNC) e o sistema cardiovascular (SCV) são sobretudo, susceptíveis a suas ações ${ }^{3}$.

Encontramos dois tipos de vasoconstritores no Brasil, a felipressina e as aminas simpatomiméticas. A felipressina é um análogo sintético da vasopressina (hormônio antidiurético), e está presente em soluções em que o sal anestésico é a prilocaína. Tem valor mínimo no controle da hemostasia, o que nos explica o sangramento maior durante procedimentos cirúrgicos em relação as que contem agentes simpatomiméticos. Já as aminas simpatomiméticas podem ou não apresentar um núcleo catecol em sua estrutura química, denominando-se respectivamente como catecolaminas ou não catecolaminas ${ }^{1}$.

Existem também, contraindicações absolutas ao uso de vasoconstritores, como no caso de angina péctoris instável, acidente vascular cerebral recente, infarto do miocárdio de até 6 meses, arritmias refratárias, revascularização miocárdia recente, hipertensão grave não tratada ou não controlada, diabete mellitus não controlado, feocromocitoma, insuficiência cardíaca congestiva intratável, hipertensão grave não tratada, hipersensibilidade a sulfitos e hipertireoidismo não controlado $^{8,11}$.

O hipertireoidismo é o aumento da produção e liberação dos hormônios tireoidianos pela glândula tireóide ${ }^{6}$. A glândula tireóide tem como função tirar o iodo da dieta e transformá-lo em T3 (triiodotiroxina) e T4 (tiroxina). Eles capturam e armazenam o iodo, os ligam nos hormônios de tirosina que formam os hormônios da tireoide e são liberados para o sangue ${ }^{5}$. É importante na regulação e no controle do índice metabólico do corpo, que quando é comprometido, abala o metabolismo dos carboidratos, proteínas e lipídios ${ }^{13,5}$.

Devido ao efeito do hormônio tireoidiano sobre o miocárdio, os pacientes portadores dessa doença frequentemente possuem hipertensão, insuficiência cardíaca e taquidisritmias atriais ${ }^{10}$.

Os efeitos do hormônio hipertireoidiano se equipara aos efeitos das catecolaminas plasmáticas, causando taquicardia, hipertensão e aumento do déficit cardíaco. Pacientes portadores de hipertireoidismo apresentam uma hipersensibilidade as catecolaminas, 
devendo assim, os anestésicos com adrenalina serem usados com parcimônia ${ }^{14}$.

O estudo tem como objetivo realizar uma revisão de literatura sobre a conduta correta, quanto ao uso de vasoconstritores em pacientes portadores de Hipertireoidismo.

\section{Materiais e Métodos}

Este estudo trata-se de uma revisão de literatura, com o objetivo de direcionar o cirurgião-dentista sobre a conduta correta, quanto ao uso de anestésicos locais com vasoconstritores, nos procedimentos em pacientes portadores de Hipertireoidismo. Para isso, foram realizadas pesquisas em bases de dados Scientific Eletronic Library Online (SciELO), Google Acadêmico e PubMed com os descritores: "Hipertireoidismo", "vasoconstritor", "anestésicos locais" e "alterações sistêmicas" além da utilização dos livros de terapêutica medicamentosa e urgências médicas em odontologia, no período de novembro a dezembro de 2018. Uma seleção de 14 documentos serviu de referência bibliográfica na elaboração desta revisão de literatura.

\section{Resultados e Discussão}

Embora o uso de anestésicos com vasoconstritor seja muito utilizado, muitas dúvidas ainda persistem em relação a pacientes com desordens sistêmicas, que influenciam no tratamento odontológico.

Uma anamnese completa é importante para se estabelecer um correto planejamento de tratamento odontológico. Isso evitaria e preveniria complicações sérias, e orientariam sobre o uso racional do anestésico local com vasoconstritor ${ }^{10,13}$.

Os sintomas do hipertireoidismo mais frequentes citados são o aumento da sudorese, intolerância ao calor, perda de peso, nervosismo, sintomas oculares como oftalmismo, aumento de apetite, fadiga, palpitações e comprometimento na capacidade de tomar decisões importantes ${ }^{1,5,10}$.

Um estudo afirma que para os cirurgiõesdentistas, a principal razão para que durante um procedimento odontológico em pacientes portadores de hipertireoidismo não controlado evite-se anestésicos locais com vasoconstritor, é a possibilidade das aminas simpaticomiméticas potencializarem o efeito vascular do hormônio da tireoide ${ }^{10}$.

Entretanto, a dor gerada quando não se obtém uma anestesia eficaz é estressante ao paciente. As catecolaminas endógenas (adrenalina e noradrenalina) liberadas durante o estresse para o sistema cardiovascular é um nível 40 vezes maior do que em repouso, o que é superior a quantidade de vasoconstritor presente em um a dois tubetes de anestésicos 9 .
É relatado um estudo onde foi realizado a aplicação de epinefrina e noreprinefrina em 5 pacientes. Não foi observado uma diferença considerável na pressão arterial sistêmica, média de pressão arterial direita, frequência cardíaca, índice cardíaco e alteração na resistência sistêmica total. Os pacientes foram avaliados em uma sessão de hipertireoidismo e uma sessão eutireroidiana ${ }^{10}$.

Outro estudo, relatado pelo mesmo autor, foi a comparação de adrenalina subcutânea e propranolol na frequência cardíaca e pressão arterial em quatro pacientes com hipertireoidismo e cinco pacientes eutireoidianos. Como resultado semelhante nesses dois grupos, houve uma falta de hipersensibilidade do sistema cardiovascular com as catecolaminas no hipertireoidismo ${ }^{10}$.

Em contrapartida, também foi apresentado alguns casos isolados da presença de hipersensibilidade com as catecolaminas nos pacientes com hipertireoidismo, e observaram também a presença de uma doença cardíaca subclínica frequentemente ligada ao hipertireoidismo, mostrando assim que é preciso ter atenção no tratamento desses pacientes, recomendando, portanto, o uso de anestésicos locais sem vasoconstritores em pacientes não controlados ${ }^{10}$.

Se tem relatos sobre o efeito das catecolaminas plasmáticas e suas reações, como a taquicardia. E seguindo essa concepção, é afirmado que pacientes portadores de hipertireoidismo apresentam hipersensibilidade as catecolaminas. Indicando o uso de anestésicos com adrenalina de forma racional ${ }^{14}$.

A utilização da adrenalina é contraindicada com vasoconstritor em pacientes com hipertireoidismo. Ocorre a sensibilização do miocárdio e há o desencadeamento de arritmias cardíacas. Nesses casos, a felipressina é o vasoconstritor mais recomendado, exceto em grávidas ${ }^{3}$.

Em um paciente portador de hipertireoidismo não controlado, não só o uso de anestésico com vasoconstritor está contraindicado, como também o próprio tratamento odontológico. Urgências devem ser solucionadas em ambiente hospitalar, com avaliação médica $^{5,1}$

Foi observado em um estudo, que os vasoconstritores associados com os anestésicos locais não produzem, normalmente, efeitos farmacológicos a não ser a constrição arteriolar localizada. $\mathrm{E}$ que, com a injeção intravascular acidental, as interferências medicamentosas e altas doses podem levar a efeitos marcantes no sistema circulatório ${ }^{12}$.

Em pacientes com a doença controlada, as soluções com epinefrina 1:100.000 ou 1:200.000 podem ser utilizadas, com o limite de dois tubetes por sessão. É preferível o uso da solução 1:100.000. Deve-se usar a menor dose possível e aspirar antes da injeção do anestésico, evitando a aplicação intravascular ${ }^{1,5}$. 


\section{Conclusão}

Os anestésicos com vasoconstritores nos trazem grandes vantagens para uma anestesia eficaz. E porisso, é necessário ter domínio sobre os fármacos e as condições sistêmicas do paciente, evitando-se complicações. Para isso, a anamnese deve ser obrigatoriamente preenchida detalhadamente.

Os hormônios hipertireoidianos são comparados aos efeitos das catecolaminas plasmáticas, causando alteração cardíacas. E diante disso, anestésicos com vasoconstritor devem ser utilizados racionalmente.

O uso de anestésico sem vasoconstritor é também citado, mas sabendo que, a dor gerada quando não se obtém uma anestesia eficaz, que normalmente é por meio do uso vasoconstritor, gera um estresse. Durante esse estresse, catecolaminas endógenas são liberadas em uma quantidade superior a existente em um a dois tubetes de anestésico.

Anestésicos com felipressina podem ser uma opção também em pacientes com hipertireoidismo controlado, sabendo que não podem ser utilizados em pacientes grávidas.

Pacientes portadores de hipertireoidismo não controlado, são contraindicados o uso dos vasoconstritores, assim como qualquer procedimento odontológico. Sugerindo ao paciente o controle da doença para a início do tratamento.

\section{Referências Bibliográficas}

1. 1. Andrade ED. Terapêutica Medicamentosa em Odontologia. Organizador: Eduardo Dias de Andrade. - 3. Ed. - São Paulo: Artes Médicas, 2014

2. Caldas CS, Bergamaschu CC, Succi GM, Motta RHL, Ramacciato JC. Clinical evaluation of diferente epinephrine concentrations for local dental anestesia. Rev Dor. São Paulo, 2015 jan-mar;16(1):1-5.

3. Carvalho RWF, Pereira CU, Anjos ED, Laureano Filho JR, Vasconcelos BCE. Anestésicos Locais: Como Escolher e Previnir Complicações Sistémicas. Rev Port Estomatol Med Dent Cir Maxilofac 2010; 51:113-20.

4. Duarte DF. Uma Breve História do Ópio e dos Opióides. Rev Bras Anestesiol 2005 Fevereiro; 55(1):135-46.

5. Lizardi PG, Jimenéz HAG. Urgências médicas em Odontologia. $2^{\mathrm{a}}$ edición. Editorial El Manual Miderno, S.a de C.V. México, 2012. p. 255-63.

6. Maia AL, Scheffel RS, Meyer ELS, Mazeto GMFS, Carvalho GA, Graf H, et al. Consenso brasileiro para o diagnóstico e tratamento do hipertireoidismo: recomendações do Departamento de Tireoide da Sociedade Brasileira de Endocrinologia e Metabologia. Arq Bras Endocrinol Metab., 2013;57(3):205-32

7. Oliveira AEM, Simone JL, Ribeiro RA. Pacientes hipertensos e a anestesia na Odontologia: devemos utilizar anestésicos locais associados ou não com vasoconstritores?. HU Revista, Juiz de Fora, 2010 jan./ mar.;36(1):69-75,

8. Paiva LCA, Cavalcanti AL. Anestésicos locais em Odontologia: Uma revisão de literatura. Publ. UEPG Ci. Biol. Saúde, Ponta Grossa, 2005 jun.;11(2):35-42.

9. Palma FR, Lins LHS, Branco FP, Wygladala LG. Verificação da variação da pressão arterial pelo uso de anestésicos locais com vasoconstritor.
Revista Odonto Ciência - Fac. Odonto/PUCRS, 2005 jan./mar.;20 (47):35-9.

10. Perusse R, Goulet JP, Turcotte JY.Contra indications to vasoconstrictors in dentistry: Part II Hyperthyroidism, diabetes, sulfite sensitivity, corticodependent asthma, and pheochromocytoma. Oral Surg Oral Med Oral Pathol, November 1992; 74:687-91.

11. Siqueira AL, Rocha FS, Carvalho QA. Uso de anestésicos locais com vasoconstritor em pacientes hipertensos. Em: Universidade Federal de Uberlândia $4^{\text {a }}$ Semana do Servidor e $5^{\text {a }}$ Semana Acadêmica 2008 - UFU 30 anos. Uberlândia, Minas Gerais, 2008. Disponível em: https://ss14799. websiteseguro.com/swge5/seg/cd2008/PDF/SA08-20430.PDF

12. Soares RG, Salles AA, Irala LED, Limongi O. Como escolher um adequado anestésico local para as diferentes situações na clínica odontológica diária? RSBO, 2006 ;3(1):35-40.

13. Souza, M. O. F. de et al. Incidência de alterações sistêmicas e uso de medicamentos em pacientes atendidos em clínica odontológica. ConScientiae Saúde, São Paulo, 2007;6(2):305-11.

14. Vasconcellos RJH, Nogueira RVB, Leal AKR, Oliveira CTV, Bezerra JGB. Alterações Sistêmicas Decorrentes do Uso de Lidocaína e Prilocaína na Prática Odontológica. Rev. Cir. Traumat. Buco Maxilo-Facial, 2002 jan/ jun.;1(2):13-9. 